Pacific Journal of Mathematics

A CLASS OF BISIMPLE INVERSE SEMIGROUPS 


\title{
A CLASS OF BISIMPLE INVERSE SEMIGROUPS ${ }^{1}$
}

\author{
R. J. WARNE
}

The purpose of this paper is to study a certain generalization of the bicyclic semigroup and to determine the structure of some classes of bisimple (inverse) semigroups mod groups.

Let $S$ be a bisimple semigroup and let $E_{S}$ denote the collection of idempotents of $S . E_{S}$ is said to be integrally ordered if under its natural order it is order isomorphic to $I^{0}$, the nonnegative integers, under the reverse of their usual order. $E_{S}$ is lexicographically ordered if it is order isomorphic to $I^{0} \times I^{0}$ under the order $(n, m)<(k, s)$ if $k<n$ or $k=n$ and $s<m$. If $\mathscr{H}$ is Green's relation and $E_{S}$ is lexicographically ordered, $S / \mathscr{H} \cong\left(I^{0}\right)^{4}$ under a simple multiplication. A generalization of this result is given to the case where $E_{S}$ is $n$ lexicographically ordered. The structure of $S$ such that $E_{S}$ is integrally ordered and the structure of a class of $S$ such that $E_{S}$ is lexicographically ordered are determimed mod groups. These constructions are special cases of a construction previously given by the author. This paper initiates a series of papers which take a first step beyond the Rees theorem in the structure theory of bisimple semigroups.

The theory of bisimple inverse semigroups has been investigated by Clifford [2] and Warne [7], [8], and [9].

If $S$ is a bisimple semigroup such that $E_{S}$ is lexicographically ordered, $S / \mathscr{H}$ is shown to be isomorphic to the semigroup obtained by embedding the bicyclic semigroup $C$ in a simple semigroup with identity by means of the Bruck construction [1]. We denote this semigroup by $\mathrm{CoC}$. An interpretation of this construction introduced by the author in [10] is used.

In [2, p. 548, main theorem], Clifford showed that $S$ is a bisimple inverse semigroup with identity if and only if $S \cong\{(a, b): a, b \in P\}$, where $P$ is a certain right cancellative semigroup with identity isomorphic to the right unit subsemigroup of $S$, under a suitable multiplication and definition of equality. In the special case $\mathscr{L}$ (Green's relation) is a congruence on $P$ (equivalently, $\mathscr{C}$ is a congruence on $S$ ), Warne showed [8, p. 1117, Theorem 2.1; p. 1118, Theorem 2.2 and first remark] that $P \cong U \times P / \mathscr{L}$, where $U$ is the group of units of $P$ (of $S$ ), under a Schreier multiplication or equivalently, $S \cong$ $\{((a, b),(c, d)): a, c \in U, b, d \in P / \mathscr{L}\}$. Warne also notes [8, p. 1118, second remark and p. 1121, Example 2] that a class of semigroups

1 Some of the results given here have been stated in a research announcement in the Bull. Amer. Math. Soc. [12]. 
studied by Rees [6, p. 108, Theorem 3.3] may be substituted as a class of $P$ in the above construction (here, $P / \mathscr{P} \cong\left(I^{0},+\right),[8, \mathrm{p}$. 1118, Equation 2.9]). By [2, p. 553, Theorem 3.1], this substitution will yield the multiplication for the class of bisimple (inverse with identity) semigroups such that $E_{S}$ is integrally ordered in terms of ordered quadruples. We carry out the indicated calculations, which are routine, in detail here to yield Equation 3.4, which with the equality definition $((g, n),(h, m))=\left(\left(g_{1}, n_{1}\right),\left(h_{1}, m_{1}\right)\right)$ if $g g_{1}^{-1}=h h_{1}^{-1}, n=n_{1}$ and $m=m_{1}$, is the structure theorem in terms of ordered quadruples. (The author was aware of this result in the spring of 1963.)

N. R. Reilly informed us he had a multiplication for these semigroups $(*$, p. 572) in terms of ordered triples. His elegant formulation follows from our quadruple formulation by an application of [2, p. 548, Equation 1.2]. A still more convenient formulation is $S \cong U \times C$ with a suitable multiplication. ${ }^{2}$

Next, it is shown that for a class of bisimple semigroups $S$ such that $E_{S}$ is lexicographically ordered, $S \cong G X(C o C)$, where $G$ is a certain group, under a suitable multiplication. The above techniques of [8] are again utilized here. The greater generality achieved in the integrally ordered case appears to arise from the fact that in this case $P$ is a splitting extension of $U$ by $I^{0}$ (i.e., in notation of $[8$, p. 1117], $a^{b}=e$, the identity of $U$ for all $\left.a, b \in I^{0}\right) .^{3}$

These structure theorems resemble the Rees theorem for completely simple semigroups [3] in that they completely describe the structure or certain classes of bisimple semigroups mod groups.

$\mathscr{R}, \mathscr{L}, \mathscr{H}$, and $\mathscr{D}$ will denote Green's relations [3, p. 47]. $R_{a}$ denotes the equivalence class containing the element $a$. Unless otherwise stated, the definitions and terminology of [3] will be used.

1. Preliminary discussion. We first summarize the construction of Clifford referred to in the introduction.

Let $S$ be a bisimple inverse semigroup with identity. Such semigroups are characterized by the following conditions $[8, \mathrm{p} .1111 ; 3,4,2$ are used].

A1: $S$ is bisimple.

A2: $S$ has an identity element.

A3: Any two idempotents of $S$ commute.

It is shown by Clifford [2] that the structure of $S$ is determined by that of its right unit semigroup $P$ and that $P$ has the following properties:

B1: The right cancellation law holds in $P$.

B2: $P$ has an identity element

\footnotetext{
2 See p. 576, (2).

3 See p. 576, (3), (5).
} 
B3: The intersection of two principal left ideals of $P$ is a principal left ideal of $P$.

Let $P$ be any semigroup satisfying B1, B2 and B3. From each class of $\mathscr{L}$-equivalent elements of $P$, let us pick a fixed representative. B3 states that if $a$ and $b$ are elements of $P$, there exists $c$ in $P$ such that $P a \cap P b=P c . \quad c$ is determined by $a$ and $b$ to within $\mathscr{L}$-equivalence. We define $a v b$ to be the representative of the class to which $c$ belongs. We observe also that

$$
a \vee b=b \vee a
$$

We define a binary operation * by

$$
(a * b) b=a \vee b
$$

for each pair of elements $a, b$ of $P$.

Now let $P^{-1} o P$ denote the set of ordered pairs $(a, b)$ of elements of $P$ with quality defined by

$$
(a, b)=\left(a^{\prime}, b^{\prime}\right) \text { if } a^{\prime}=u a \text { and } b^{\prime}=u b \text { where } u \text { is a }
$$
unit in $P$ ( $u$ has a two sided inverse with respect to 1 , the identity of $P$ ) .

We define product in $P^{-1} o P$ by

$$
(a, b)(c, d)=((c * b) a,(b * c) d) .
$$

Clifford's main theorem states: Starting with a semigroup $P$ satisfying B1, 2, 3, Equations (1.2), (1.3), and (1.4) define a semigroup $P^{-1} o P$ satisfying A1, 2, 3. $P$ is isomorphic with the right unit subsemigroup of $P^{-1} o P$ (the right unit subsemigroup of $P^{-1} o P$ is the set of elements of $P^{-1} o P$ having a right inverse with respect to 1 ; this set is easily shown to be a semigroup). Conversely, if $S$ is a semigroup satisfying A1, 2, 3, its right unit subsemigroup $P$ satisfies $\mathrm{B} 1,2,3$ and $S$ is isomorphic to $P^{-1} O P$.

The following results are also obtained:

Lemma 1.1 [2]. For $a, b$ in $P$ and $u, v$ in $U$, the group of units of $P$, we have $(u a * v b) v=a * b$. The unit group of $P$ is equal to the

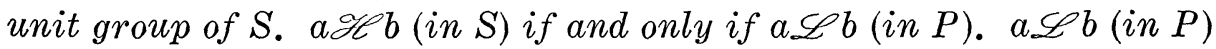
if and only if $a=u b$ for some $u$ in $U$.

THEOREM 1.1 [2]. Let $S$ be a semigroup satisfying A1, 2, 3, and let $P$ be its right unit subsemigroup. Then $P$ satisfies B3 (as well as B1 and B2), and the semi-lattice of principal left ideals of $P$ under intersection is isomorphic with the semi-lattice of idempotent 
elements of $S$.

We now briefly review the work of Rédei [5] on the Schreier extension theory for semigroups (we actually give the right-left dual of his construction) and we also present some pertinent material from [8]. Let $G$ be a semigroup with identity $e$. We consider a congruence relation $\rho$ on $G$ and call the corresponding division of $G$ into congruence classes a compatible class division of $G$. The class $H$ containing the identity is said to be the main class of the division. $H$ is easily shown to be a subsemigroup of $G$. The division is called right normal if and only if the classes are of the form,

$$
H a_{1}, H a_{2}, \cdots\left(a_{1}=e\right)
$$

and $h_{1} a_{i}=h_{2} a_{i}$ with $h_{1}, h_{2}$ in $H$ implies $h_{1}=h_{2}$. The system (1.5) is shown to be uniquely determined by $H . H$ is then called a right normal divisor of $G$ and $G / \rho$ is denoted by $G / H$.

Let $G, H$, and $S$ be semigroups with identity. Then, if there exists a right normal divisor $H^{\prime}$ of $G$ such that $H \cong H^{\prime}$ and $S \cong$ $G / H^{\prime}, G$ is said to be a Schreier extension of $H$ by $S$.

Now, let $H$ and $S$ be semigroups with identities $E$ and $e$ respectively. Consider $H X S$ under the following multiplication:

$$
\begin{aligned}
(A, a)(B, b)= & \left(A B^{a} a^{b}, a b\right)(A, B \text { in } H ; a, b \text { in } S) \\
& a^{b}, B^{a}(\text { in } H)
\end{aligned}
$$

designate functions of the arguments $a, b$ and $B, a$ respectively, and are subject to the conditions

$$
a^{e}=E, e^{a}=E, B^{e}=B, E^{a}=E .
$$

We call $H \times S$ under this multiplication a Schreier product of $H$ and $S$ and denote it by $H_{0} S$.

Rédei's main theorem states:

THEOREM 1.2 (Redei). A Schreier product $G=H o S$ is a semigroup if and only if

$$
\begin{aligned}
(A B)^{c} & =A^{c} B^{c}(A, B \text { in } H: c \text { in } S) \\
\left(B^{a}\right)^{c} c^{a} & =c^{a} B^{c a}(B \text { in } H ; a, c \text { in } S) \\
\left(a^{b}\right)^{c} c^{a b} & =c^{a}(c a)^{b}(a, b, c \text { in } S)
\end{aligned}
$$

are valid. These semigroups (up to an isomorphism) are all the Schreier extensions of $H$ by $S$ and indeed the elements $(A, e)$ form a right normal divisor $H^{\prime}$ of $G$ for which 


$$
\begin{aligned}
G / H^{\prime} & \cong S\left(H^{\prime}(E, a) \rightarrow a\right) \\
H^{\prime} & \cong H((A, e) \rightarrow A)
\end{aligned}
$$

are valid.

Theorem 1.3 [8]. Let $U$ be a group with identity $E$ and let $S$ be a semigroup satisfying B1 and B2 (denote its identity by e) and suppose $S$ has a trivial group of units. Then every Schreier ex-

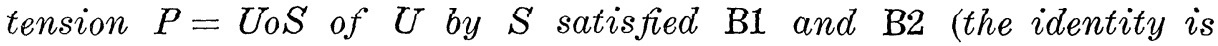
$(E, e)$ ) and the group of units of $P$ is $U^{\prime} \cong\{(A, e): A$ in $U\} \cong U$ Furthermore $\mathscr{L}$ is a congruence relation on $P$ and $P / \mathscr{L} \cong S . P$ satisfies B3 if and only if $S$ satisfies B3.

Conversely, let $P$ be a semigroup satisfying $\mathrm{B} 1$ and $\mathrm{B} 2$ on which $\mathscr{L}$ is a congruence relation. Let $U$ be the group of units of $P$. Then $U$ is a right normal divisor of $P$ and $P / U \cong P / \mathscr{L}$. Thus, $P$ is a Schreier extension of $U$ by $P / \mathscr{L} . P / \mathscr{L}$ satisfies $\mathrm{B} 1$ and $\mathrm{B} 2$ and has a trivial group of units.

The following statements are valid for any semigroup obeying the conditions of Theorem 1.3 (i.e. semigroups satisfying B1, B2 on which $\mathscr{L}$ is a congruence).

$$
\begin{aligned}
& P(A, a)=\{(C, b a): C \text { in } U, b \text { in } S\} . \\
& (A, a) L(B, b) \text { if and only if } a=b .
\end{aligned}
$$

As remarked in [8], the semigroups considered by Rees (Theorem 1.5 below) fall into this category.

Now, Rees defines a right normal divisor in a different manner than Rédei. He says that $V$ is a right normal divisor of a semigroup $P$ satisfying $\mathrm{B} 1$ and B2 if $V$ is a subgroup of the unit group $U$ of $P$ and $a U \subseteq U a$ for all $a$ in $P$. However, let us show that the Rees definition is just a specialization of the Rédei definition to the case where the main class is a group and the semigroup we are dealing with satisfies $\mathrm{B} 1$ and $\mathrm{B} 2$. In this case, suppose that $V$ is a right normal divisor in the sense of Rédei. Then, clearly, $V$ is a subgroup of $U$. The congruence class containing $a$ is just $V a$. Let $u$ in $V$.

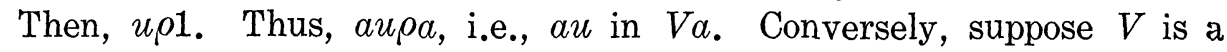
right normal divisor in the sense of Rees. Let us define $a \rho b$ if and only if $V a=V b$. It is easily seen that $\rho$ is a congruence on $P$ with main class $V$, i.e., $V$ is a right normal divisor in the sense of Rédei.

Let us now briefly review the theory of Rees [6]. Let $P$ be a semigroup satisfying $\mathrm{B} 1$ and $\mathrm{B} 2$. The partially ordered system of principal left ideals of $P$, ordered by inclusion, will be denoted by $O(P)$ and termed the ideal structure of $P$. If $(O, \geqq)$ is a partially 
ordered set, we denote the set of all elements $x$ of $O$ satisfying $x \leqq a$ by $O_{a}$ and term such a set a section of $O$. Then we take as $P(O)$ the set of all order isomorphic mappings $\gamma$ of $O(P)$ onto sections of $O(P)$. If $U$ is the group of units of $S, M=(g$ in $U / x g$ in $U x$ for all $x$ in $P$ ) is the greatest right normal divisor of $P$.

The following theorems are established.

THeOREM 1.4 [6]. If $P$ has an ideal structure $O(P)$ and $M$ is the right normal divisor just described, then there is a subsemigroup $P^{\prime}$ of $P(O)$ isomorphic to $P / M$. Further, every principal left ideal of $P(O)$ has a generator in $P^{\prime}$.

THEOREM 1.5 [6]. A semigroup $P$ satisfying B1 and B2 whose ideal structure is isomorphic with $\vartheta$ (the ideal structure of $\left(I^{0},+\right)$ ) and whose group of units is isomorphic with a given group $G$ is isomorphic with a semigroup $T=G \times I^{0}$ under the following multiplication (1.14) $(g, m)(h, n)=\left(g\left(h \alpha^{m}\right), m+n\right), g, h$ in $G, m, n$ in $I^{0}, \alpha$ being an endomorphism of $G, \alpha^{0}$ being interpreted as the identity transformation of $G$ and conversely $T$ has the above properties.

LEMMA 1.2. Let $S$ be a bisimple inverse semigroup with identity with right unit subsemigroup $P$. $U$, the group of units of $P$, is a right normal divisor of $P$ if and only if $\mathscr{H}$ is a congruence on $S$.

Proof. Let $U$ be a right normal divisor of $P$. Let $(a, b),(c, d)$ be in $S$ and suppose that $(a, b) \mathscr{H}(c, d)$. Now $(a, b) \mathscr{R}(c, d)$ if and only if $a=u c$ where $u$ in $U$ and $(a, b) \mathscr{L}(c, d)$ if and only if $b=v d$ where $v$ in $U$. I will prove the first. Suppose that $(a, b) \mathscr{R}(c, d)$. Then there exists $(x, y),(w, z)$ in $S$ such that $(a, b)=(c, d)(x, y)$ and $(c, d)=(a, b)(w, z)$. Thus, by 1.3 and $1.4 a=p(x * d) c$ and $c=q(w * b) a$ where $p, q$ in $U$. Thus, by B1 and B2 $a=u c$ for some $u$ in $U$ by $\mathrm{B} 1$ and B2. Now suppose that $a=u^{\prime} c$ for some $u^{\prime}$ in $U$. We note first that $(b * b) b=b \vee b=u b$ for some $u$ in $U$ by 1.2, the definition of $\vee$, and Lemma 1.1. Thus, $b^{*} b=u$ by B1.

Now $(a, b)\left(b, u^{\prime} d\right)=\left(u a, u u^{\prime} d\right)=\left(u^{\prime-1} a, d\right)=(c, d)$ by (1.3). Similarly $(c, d)\left(d, u^{\prime-1} b\right)=(a, b)$, i.e., $(a, b) \mathscr{R}(c d)$.

Let $(p, q)$ be in $S$. Then by (1.4),

$$
\begin{aligned}
& (a, b)(p, q)=((p * b) a,(b * p) q) \\
& (c, d)(p, q)=((p * d) c,(d * p) q)
\end{aligned}
$$

Since $(a, b) \mathscr{H}(c, d)$ there exists $u, v$ in $U$ such that $a=u c, b=v d$. Thus, by Lemma 1.1 and the fact that $U$ is a right normal divisor

$$
(p * b) a=(p * v d) u c=(1 p * v d) v v^{-1} u c=(p * d) v^{-1} u c=t(p * d) c,
$$


where $t$ is in $U$.

Thus, $(a, b)(p, q) \mathscr{R}(c, d)(p, q)$ and $\mathscr{R}$ is a right congruence. Since $\mathscr{R}$ is always a left congruence, it is a congruence. One shows similarly that $\mathscr{L}$ is a congruence. Thus, $\mathscr{H}$ is a congruence relation on $S$.

Suppose $\mathscr{H}$ is a congruence on $S$. Let $a, b$ in $P$ and suppose $a \mathscr{L} b$ (in $P$ ). By Lemma $1.1 a \mathscr{H} b$ (in $S$ ). Thus $c$ in $P$ implies $c a \mathscr{H} c b$ (in $S$ ) and $c a \mathscr{L} c b$ (in $P$ ) by Lemma 1.1. Hence $\mathscr{L}$ is a congruence on $P$ and $U$ is a right normal divisor of $P$ by Theorem 1.3.

2. The Bruck product. Let $S$ be an arbitrary semigroup and $C$ be the bicyclic semigroup ([3], p. 43), i.e., $C$ is the set of all pairs of nonnegative integers with multiplication given by $(m, n)\left(m^{\prime}, n^{\prime}\right)=$ $\left(m+m^{\prime}-\min \left(n, m^{\prime}\right), n+n^{\prime}-\min \left(n, m^{\prime}\right)\right)$. Consider $W=C \times S$ with multiplication given by $((m, n), s)\left(\left(m^{\prime}, n^{\prime}\right), s^{\prime}\right)=\left((m, n)\left(m^{\prime}, n^{\prime}\right), f\left(n, m^{\prime}\right)\right)$ where $f\left(n, m^{\prime}\right)=s, s s^{\prime}$, or $s^{\prime}$ according to whether $n>m^{\prime}, n=m^{\prime}$, or $n<m^{\prime}$. We call $W$ the Bruck product of $C$ and $S$ and write $W=$ CoS. I used a special case of this product in [10]. CoC is easily shown to be a bisimple inverse semigroup with identity for which $E_{S}$ is lexicographically ordered. If $S$ is an arbitrary semigroup, let $S^{1}$ be $S$ with an appended identity [3, p. 4]. One can show that $C_{0} S^{1}$ is a simple semigroup with identity containing $S$ as a subsemigroup. Since this is equivalent to the construction of R. H. Bruck [1] for embedding an arbitrary semigroup in a simple semigroup with identity, we call $o$ a Bruck product.

THEOREM 2.1 [8]. Let $S$ and $S^{*}$ be bisimple inverse semigroups with identity with right unit subsemigroups $P$ and $P^{*}$ respectively. $S \cong S^{*}$ if and only if $P \cong P^{*}$.

THEOREM 2.2. Let $S$ be a bisimple (inverse) semigroup. $E_{S}$ is lexicographically ordered if and only if $\mathscr{H}$ is a congruence on $S$ and $S / \mathscr{H} \cong C o C$ where $C o C$ denotes the Bruck product of $C$ by $C$.

Proof. First we suppose that $E_{s}$ is lexicographically ordered. Clearly $S$ has an identity. For let $e$ be the largest element of the lexicographic chain. If $a$ in $S, a$ is in $R_{f}$ for some $f$ in $E_{S}$ since $S$ is regular. Then, $e a=e f a=f a=a$. Similarly, $a e=a$. Let $P$ be the right unit subsemigroup of $S$. Then by Theorem 1.1, we may write the ideal structure of $P, O(P)$ as follows:

$$
\begin{aligned}
& (0,0)>(0,1)>(0,2)>(0,3)> \\
& (1,0)>(1,1)>(1,2)>(1,3)> \\
& (2,0)>(2,1)>(2,2)>(2,3)>
\end{aligned}
$$




$$
\begin{aligned}
& (3,0)>(3,1)>(3,2)>(3,3)> \\
& (4,0)>(4,1)>(4,2)>(4,3)>
\end{aligned}
$$

If we define for $(m, k)$ in $O(P)$

$$
\begin{aligned}
(n, s) t_{(m, k)}= & (n+m, s) \text { if } n>0 \\
& (m, s+k) \text { if } n=0
\end{aligned}
$$

we easily see that $t_{(m, k)}$ is an order isomorphism of $O(P)$ onto the section of $O(P)$ determined by $(m, k)$. In fact all order isomorphisms of $O(P)$ onto sections of $O(P)$ are of this form.

Clearly $P(O) \cong I^{0} X I^{0}$ under the multiplication

$$
\begin{aligned}
(n, s)(m, k)= & (n+m, s) \text { if } n>0 \\
& (m, s+k) \text { if } n=0 .
\end{aligned}
$$

Thus, the only subsemigroup of $P(O)$ containing a generator of every principal left ideal of $P(O)$ is $P(O)$ itself. This follows since $P(O)(n, k)=\left((u+n, v): u, v\right.$ in $\left.I^{0}, u>0\right) U\left((n, v+k): v\right.$ in $\left.I^{0}\right)$. The unit group of $P(O)$ is trivial (note the identity of $P(O)$ is $(0,0)$ ).

By Theorem 1.4, $P / M \cong P(O)$. Since the unit group of $P(O)$ is trivial, $M=U$. Thus, again by Theorem 1.4, $U$ is a right normal divisor of $P$. Thus, $\mathscr{C}$ is a congruence on $S$ by Lemma 1.2. Since ([8], p. 1111) any homomorphic image of a bisimple inverse semigroup with identity is a bisimple inverse semigroup with identity, $S / \mathscr{H}$ is such a semigroup.

Let $a \rightarrow \bar{a}$ denote the natural homomorphism of $S$ onto $S / \mathscr{H}$. If $\bar{a}$ is a right unit of $S / \mathscr{H}$ there exists $\bar{x}$ in $S / \mathscr{H}$ such that $\bar{a} \bar{x}=\overline{1}$, where 1 is the identity of $S$. Thus, ax $\mathscr{H} 1$ and there exists $y$ in $S$ such that $a x y=1$, i.e., $a$ in $P$. Now, if $a$ in $P, a x=1$ for some $x$ in $S$. Thus, $\bar{a} \bar{x}=1$ and $\bar{a}$ is in the right unit subsemigroup of $S / \mathscr{H}$. Hence the right unit subsemigroup of $S / \mathscr{H}$ is $P / \mathscr{H}=P / \mathscr{L} \cong P(O)$ by Lemma 1.1. Now, as noted above $C o C$ is a bisimple inverse semigroup with identity. It is easily seen that the right unit subsemigroup of $C o C$ is isomorphic to $P(O)$. Thus, by Theorem $2.1 S / \mathscr{H} \cong C o C$. The converse is clear.

COROLLARY 2.1. $S$ is a bisimple (inverse) semigroup with trivial unit group and $E_{S}$ is lexicographically ordered if and only if $S$ is isomorphic to $\mathrm{CoC}$.

Proof. This follows from Theorem 2.3 of [3]. 
LeMma 2.1. Let $S$ be a bisimple (inverse) semigroup. $E_{S}$ is integrally ordered if and only if $\mathscr{H}$ is a congruence on $S$ and $S / \mathscr{H} \cong C$.

Proof. $\mathscr{H}$ is a congruence on $S$ by ([8], p. 1118) and Lemma 1.2. By Theorem 1.1, Theorem 1.5, 1.14, and $1.13, P / \mathscr{L} \cong I^{0}$, where $I^{0}$ is the nonnegative integers under addition. But, as above, $P / \mathscr{L}$ is the right unit subsemigroup of $S / \mathscr{H C}$. Hence $S / \mathscr{H} \cong C$ by Theorem 2.1. The converse is clear.

LEMMA 2.2. $S$ is a bisimple (inverse) semigroup with trivial unit group and $E_{S}$ integrally ordered if and only if $S \cong C$.

Let $S$ be a semigroup. We say $E_{S}$ is $n$-lexicographically ordered if and only if $E_{S}$ is order isomorphic to $\underbrace{I^{0} \times I^{0} \times x I^{0}}_{n \text { times }}$ under the order

$$
\left(k_{1}, k_{2}, \cdots, k_{n}\right)<\left(s_{1}, s_{2}, \cdots, s_{n}\right)
$$

if $k_{1}>s_{1}$ or $k_{1}=s_{1}, k_{2}>s_{2}$ or $k_{i}=s_{i}(i=1,2, j-1), k_{j}>s_{j}$ or $k_{i}=$ $s_{i}(i=1,2, n-1), k_{n}>s_{n} . \quad E_{S}$ is 2-lexicographically ordered if and only if $E_{S}$ is lexicographically ordered. $E_{S}$ is 1-lexicographically ordered if and only if $E_{S}$ is integrally ordered.

We will define the $n$-dimensional bicyclic semigroup $C_{n}$ as follows: $C_{1}=C$ and $C_{n}=\left(C_{o} \cdots o(C o(C o(C o C))) \quad\right.$ for $n>1$ where $o$ is the Bruck product (there are $n-1 o$ 's).

$C_{n}$ is a bisimple inverse semigroup with $E_{\sigma_{n}} n$-lexicographically ordered. The 1-dimensional bicyclic semigroup is the bicyclic semigroup. The 2-dimensional bicyclic semigroup is the Bruck product $\mathrm{CoC}$ of $C$ and $C$.

The following theorem and corollary are obtained by employing the techniques used in the proofs of Theorem 2.1 and Corollary 2.1 respectively.

THEOREM 2.3. $S$ is a bisimple (inverse) semigroup with $E_{S}$ n-lexicographically ordered if and only if $\mathscr{H}$ is a congruence on $S$ and $S / \mathscr{H} \cong C_{n}$.

COROLlaRY 2.2. $S$ is a bisimple (inverse) semigroup with $E_{S}$ n-lexicographically ordered and trivial unit group if and only if $S \cong C_{n}$.

3. Multiplications on two classes of bisimple inverse semigroups. 
THEOREM 3.1. $S$ is a bisimple (inverse) semigroup such that $E_{S}$ is integrally ordered if and only if $S \cong G \times C$ where $G$ is a group and $C$ is the bicyclic semigroup under the multiplication:

$$
(z, n, m)\left(z^{\prime}, n_{1}, m_{1}\right)=\left(z \alpha^{n_{1}-r} z^{\prime} \alpha^{m-r},(n, m)\left(n_{1}, m_{1}\right)\right)
$$

where $r=\min \left(m, n_{1}\right), \alpha$ an endomorphism of $G, \alpha^{0}$ is the identity transformation of $G$ and juxtaposition is multiplication in $G$ and $C$.

Proof. As in the proof of Theorem 1.7, $S$ is a bisimple inverse semigroup with identity. By Theorem 1.1, Cliffords's main theorem, and Theorem 1.5, $P \cong U \times I^{0}$ where $U$ is the group of units of $S$ under the multiplication 1.14 if and only if $E_{S}$ is integrally ordered. The $\mathscr{L}$-classes of $P$ are $L_{0}, L_{1}, L_{2} \cdots L_{n} \cdots$ where $L_{n}=((g, n): g$ in $U)$ by 1.13. Let $(e, n)$ where $e$ is the identity of $U$ be a representative element of $L_{n}$. Thus, $(e, n) \vee(e, m)=(e, \max (n, m))$ by 1.12 and the definition of $\vee$. Using (1.2) by a routine calculation, we have

$$
\begin{aligned}
(e, n) *(e, m) & =(e, n-m) & & \text { if } n \geqq m \\
& =(e, o) & & \text { if } m \geqq n
\end{aligned}
$$

Using Lemma 1.1, (1.14), and Theorem 1.3, we obtain

$$
\begin{array}{rlrl}
(g, n) *(h, m)= & \left(h^{-1} \alpha^{n-m}, n-m\right) \text { if } n \geqq m \\
& \left(h^{-1}, o\right) & \text { if } m \geqq n
\end{array}
$$

Now using (1.14) (1.4), and (3.3), we obtain

$$
\begin{aligned}
& ((g, n),(h, m))\left(\left(g_{1}, n_{1}\right),\left(h_{1}, m_{1}\right)\right) \\
& \quad=\left(\left(h^{-1} g\right) \alpha^{n_{1}-r}, n_{1}+n-r,\left(g_{1}^{-1} h_{1}\right) \alpha^{m-r}, m+m_{1}-r\right) .
\end{aligned}
$$

Now, by (1.3) and (3.4), we have

$$
\begin{aligned}
& \left(e, n, g^{-1} h, m\right)\left(e, n_{1}, g_{1}^{-1} h_{1}, m_{1}\right) \\
& \quad=\left(e, n_{1}+n-r,\left(g^{-1} h\right) \alpha^{n_{1}-r}\left(g_{1}^{-1} h_{1}\right) \alpha^{m-r}, m+m_{1}-r\right)
\end{aligned}
$$

Let $z=g^{-1} h$ and $z^{\prime}=g_{1}^{-1} h_{1}$. Then

$$
*(n, z, m)\left(n_{1}, z^{\prime}, m_{1}\right)=\left(n+n_{1}-r z \alpha^{n_{1}-r}, z^{\prime} \alpha^{m-r}, m+m_{1}-r\right)
$$

or

$$
(z, n, m)\left(z^{\prime}, n_{1}, m_{1}\right)=\left(z \alpha^{n_{1}-r} z^{\prime} \alpha^{m-r},(n, m)\left(n_{1}, m_{1}\right)\right) .
$$

The converse follows by Cliffords theorem.

To actually determine the multiplication on $S$, one determines $P$ (we are actually given $P$ here) and then places $P$ in the Clifford construction. However, after one ascertains the multiplication, a very short proof of the fact can be given by the use of Theorem 1.6. 
Alternative proof of Theorem 2.1. Let $S^{*}=G \times C$ be a groupoid with multiplication (3.1). We can show that $S^{*}$ is a bisimple inverse semigroup with identity by routine calculation (we must go through this to prove the converse anyway). It is easily seen that the right unit subsemigroup $P^{*}$ of $S^{*}$ is isomorphic to $P$. Thus, $S \cong S^{*}$ by Theorem 2.1.

A semigroup with zero, 0 , is said to be 0-right cancellative if $a, b, c$ in $S, c \neq 0, a c=b c$ implies that $a=b$. If $G$ is a group, let $\varepsilon(G)$ denote the semigroup of endomorphisms of $G$.

A nontrivial group $G$ is said to be a *-group if

(1) Every nontrivial endomorphism of $G$ maps $G$ onto $G$.

(2) $\varepsilon(G)$ is 0 -right cancellative. $\left((1) \rightarrow(2)\right.$ if $G$ is an abelian group). ${ }^{4}$ The *-groups include all cyclic groups of prime order, all groups of type $p^{\infty}$, and the additive group of rational numbers. ${ }^{5}$

If $S$ is a semigroup with identity 1 and $a, x$ in $S$ with $a x=1$, we write $x=a^{-1}$.

THEOREM 3.2. $S$ is a bisimple (inverse) semigroup such that (1) $E_{s}$ is lexicographically ordered, (2) $U$ is $a^{*}$-group, (3) $a a^{-1}=1$ implies that $U a \cong a U$, if and only if $S \cong G X(C o C)$ where $G$ is a ${ }^{*}$-group, $C$ is the bicyclic semigroup, o is the Bruck product, with the multiplication,

$$
\begin{aligned}
(g,(n, k),(m, l))\left(h,\left(n_{1}, k_{1}\right),\left(m_{1}, l_{1}\right)\right) \\
=\left(g \alpha^{n_{1}-r} h \alpha^{k-r},((n, k),(m, l))\left(\left(n_{1}, k_{1}\right),\left(m_{1}, l_{1}\right)\right)\right.
\end{aligned}
$$

where $r=\min \left(n_{1}, k\right)$ and $\alpha$ is a nontrivial endomorphism of $G \alpha^{0}$ denotes the identity transformation, and juxtaposition denotes multiplication in $G$ and $C o C$.

Proof. Let $P$ be the right unit subsemigroup of $S$. If $U$ is a right normal divisor of $P$, then clearly $\mathscr{L}$ is a congruence on $P$. Thus by Theorem 2.2 Lemma 1.2, and Theorem 1.3, $P$ is a Schreier extension of $U$ by $P / U(=P / \mathscr{L})$. Now, the semigroup of right units $P^{*}$ of $\mathrm{CoC}$ is easily seen to be isomorphic to $I^{0} \times I^{0}$ under the multiplication

$$
\begin{aligned}
(n, m)(p, q)= & (n+p, m) \text { if } n>0 \\
& (n+p, m+q) \text { if } n=0
\end{aligned}
$$

Now $a=(1, o)$ and $b=(o, 1)$ are generators of $P^{*}$ and $a b=a$. Now, as remarked in the proof of Theorem 2.2 the right unit subsemigroup

${ }^{4}(1) \rightarrow(2)$ also if $G$ is simple or finite.

5 The *-groups also include all nontrivial finite simple groups. 
of $S / \mathscr{H} \cong C o C$ (Theorem 2.2) is $P / \mathscr{L}$. Thus, we may label the $\mathscr{L}$ classes of $P$ as $\left\{L_{(n, k)}: n, k\right.$ in $\left.I^{0}\right\}$. Now let $a^{\prime}$ in $L_{(1,0)}$ and $b^{*}$ in $L_{(0,1)}$. Thus, $a^{\prime} b^{*}=u a^{\prime}$ for some $u$ in $U$. Thus by (3) $u a^{\prime}=a^{\prime} v$ for some $v$ in $U$. Hence, $a^{\prime} b^{*}=a^{\prime} v, a^{\prime} b^{*} v^{-1}=a^{\prime}$. Let $b^{*} v^{-1}=b^{\prime}$. Now, since $U$ is a right normal divisor of $P, b^{*} v^{-1}=w b^{*}$ for some $w$ in $U$ and $b^{\prime}$ in $L_{(0,1)}$. Thus, $\left\{b^{\prime k} a^{\prime s}, k, s\right.$ in $\left.I^{0}\right\}$ form a complete system of representative elements (5) which is also a semigroup. Thus the factors $c^{d}$ of (1.6) are all equal to $E$, the identity of $U$. Thus, (1.6) becomes

$$
(A, n, k)(B, m, l)=\left(A B^{(n, k)},(n, k)(m, l)\right)
$$

where $A, B$ in $U,(n, k),(m, 1)$ in $P / \mathscr{L}$ and juxiaposition is multiplication in $U$ and $P / \mathscr{L}$. Now let $a=(1,0)$ and $b=(0,1)$, and let $e=(0,0)$, the identity of $P / \mathscr{L}$. Then $(E, a)(g, e)=(g \alpha, e)(E, a)(a$, fixed), $\alpha$ a transformation of $U$, since $U$ is a right normal divisor of $P$ and $\{(g, e): g$ in $U\}$ is isomorphic to $U$ (Theorem 1.3). Now $(E, a)(g, e)=\left(g^{a}, a\right)$ by 1.6. Hence $g^{a}=g \alpha$. Similarly, $g^{b}=g \beta$. By (1.8) $\alpha$ and $\beta$ are endomorphisms of $U$. By (1.9), $\left(g^{b}\right)^{a}=g^{a b}=g^{a}(g$ in $U)$. Thus $g \alpha=g \beta \alpha, g$ in $U$, i.e., $\alpha=\beta \alpha$. Let us first suppose that $\alpha \neq 0$ in $\varepsilon(U)$. Then since $\varepsilon(U)$ is 0 -right cancellative $\beta$ is the identity automorphism of $U$. Now, by $1.9, g^{(n, k)}=g^{(0,1)^{k}(1,0)^{n}}=\left(g^{(1,0)^{n}}\right)^{(0,1)^{k}}=$ $g \alpha^{n} \beta^{k}=g \alpha^{n}$ and (3.5) becomes

$$
(A, n, k)(B, m, l)=\left(A\left(B \alpha^{n}\right),(n, k)(m, l)\right)
$$

By routine calculation, we can show that $S^{*}=U x\left(C_{0} C\right)$ under the multiplication

$$
\begin{aligned}
& (g,(n, k),(m, l))\left(h,\left(n_{1}, k_{1}\right),\left(m_{1}, l_{1}\right)\right) \\
& =\left(g \alpha^{n_{1}-r} h \alpha^{k-r},\left(((n, k),(m, l))\left(\left(n_{1}, k_{1}\right),\left(m_{1}, l_{1}\right)\right)\right)\right),
\end{aligned}
$$

where $r=\min \left(n_{1}, k\right)$ and $\alpha$ is an endomorphism of $U$, is a bisimple inverse semigroup with identity. To show associativity is straight forward, but tedious. Now,

$$
(g,(n, k),(m, l)) \mathscr{R}\left(h,\left(n_{1}, k_{1}\right),\left(m_{1}, l_{1}\right)\right) \text { if and only if } n=n_{1} \text { and } m=m_{1}
$$

and

$(g,(n, k),(m, l)) \mathscr{L}\left(h,\left(n_{1}, k_{1}\right),\left(m_{1}, l_{1}\right)\right)$ if and only if $k=k_{1}$ and $l=l_{1}$ Thus, if

$$
\begin{aligned}
& (g,(n, k),(m, l)),(h,(u, v),(r, s)) \text { in } S^{*}, \\
& (g,(n, k),(m, l)) \mathscr{R}(g,(n, v),(m, s)) \mathscr{L}(h,(u, v),(r, s))
\end{aligned}
$$

and $S^{*}$ is bisimple. $(E,(0,0),(0,0))$ where $E$ is the identity of $U$ is the identity of $S^{*}$. 
The idempotents of $S^{*}$ are $\left\{(E,(n, n),(k, k)), n, k\right.$ in $\left.I^{0}\right\}$. It is easily seen that these commute.

Thus, $S^{*}$ is a bisimple inverse semigroup with identity, [8, p. 1111].

The right unit subsemigroup $P^{*}$ of $S^{*}$ is $\{(g, 0, n, 0, k): n, k$ in $\left.I^{0}, g \in G\right\}$. It is seen immediately that $P^{*}$ is isomorphic to $P$ and hence $S \cong S^{*}$ by Theorem 2.1. Let us give the converse of this case. Now it is quite easily seen that the unit group of $S$ is $\{g,(0,0),(0,0)\} \cong$ G. (the unit group is $\left.H_{((0,0), 0,0))}\right)$. Thus, $U$ is a ${ }^{*}$-group.

The right unit subsemigroup $P$ of $S$ is $\left\{(g, n, k): n, k\right.$ in $\left.I^{\circ}\right\}$ under the multiplication

$$
\begin{aligned}
& (g, n, k)(h, m, s)=\left(g\left(h \alpha^{n}\right), n+m, k\right) \text { if } n>0 \\
& (g, 0, k)(h, m, s)=(g h, m, k+s)
\end{aligned}
$$

Let $(g, 0,0) \varepsilon U$ and $(h, m, s), m>0$ be in $P$. Since $G$ is a *-group, there exists $g^{\prime}$ in $G$ such that $h^{-1} g h=g^{\prime} \alpha^{m}$ (since $\alpha$ is nontrivial, $\alpha^{m}$ is nontrivial) as $\varepsilon(G)$ is 0 -right cancellative). Thus

$$
(g, 0,0)(h, m, s)=(g h, m, s)=\left(h\left(g^{\prime} \alpha^{m}\right), m, s\right)=(h, m, s)\left(g^{\prime}, 0,0\right) .
$$

Next, we consider $(h, 0, m)$. Now, let $g^{\prime}=h^{-1} g h$. Then,

$$
(g, 0,0)(h, 0, m)=(g h, 0, m)=\left(h g^{\prime}, 0, m\right)=(h, 0, m)\left(g^{\prime}, 0,0\right)
$$

Hence, $U$ satisfies (3).

$$
E_{S}=\left\{E,(n, n),(k, k): n, k \text { in } I^{0}\right\}
$$

and multiplication in $E_{S}$ is given by

$$
\begin{aligned}
(n, k)(m, l) & =(n, k) \text { if } n>m \\
& =(n, k) \text { if } n=m \text { and } k>l .
\end{aligned}
$$

Thus (1) is satisfied.

Next, suppose $\alpha$ is the zero of $\varepsilon(U)$, i.e., $g \alpha=E, g$ in $U$. This means $g^{\alpha}=E, g$ in $U$. Now $g^{(n, k)}=g^{(0,1)^{k}(1,0)^{n}}=\left(g^{(1,0)^{n}}\right)^{(0,1)^{k}}=(E)^{(0,1)^{k}}=E$ if $n \neq 0$. If $n=0, g^{(n, k)}=g^{(0, k)}=g \beta^{k}$. Thus, our multiplication (3.5) becomes $(A, n, k)(B, m, s)=(A, n+m, k)$ if $n \neq 0$,

$$
\left.(A, 0, k)(B, m, s)=\left(A\left(B \beta^{k}\right), m, k+s\right)\right) .
$$

Now, by $(3)$, if $(g, 0,0)$ in $U$, there exists $\left(g^{\prime}, 0,0\right)$ in $U$ such that if $m \neq 0$

$$
(g, 0,0)(B, m, s)=(g B, m, s)=(B, m, s)\left(g^{\prime}, 0,0\right)=(B, m, s) .
$$

Hence, $g B=B$ and $g=E$. Since $g$ was arbitrary, $U$ is a trivial group and we have a contradiction. Thus $\alpha$ cannot be a trivial endomorphism. 
ExAmPle. Let $G$ be $a^{*}$-group, $C$ be the bicyclic semigroup, and $o$ be the Bruck product. If we let $\alpha$ be the trivial endomorphism of $G$ in the 1-dimensional (2-dimensional) case, $S$ is a bisimple inverse semigroup with $E_{S}$ integrally (lexicographically) ordered and with group of units a *-group. However (3) of Theorem (3.2) is not satisfied. $S=C o G$ is the 1-dimensional case.

Added in proof. (1) A nontrivial group is called an e-group if every nontrivial endomorphism of $G$ is an epimorphism. The following theorem has a proof similar to that of Theorem 3.2.

THEOREM. In Theorem 3.2, replace *-group by e-group and the multiplication given there by

$$
\begin{aligned}
& (g,(n, k),(m, 1))(h,(r, s),(u, v)) \\
& \quad=\left(g \alpha^{r-\delta} \beta^{u-\gamma_{1}(r, k)} h \alpha^{k-\delta} \beta^{1-\gamma_{2}(r, k)},((n, k),(m, 1))((r, s),(u, v))\right)
\end{aligned}
$$

where if $r>k, \gamma_{1}(r, k)=0, \gamma_{2}(r, k)=1$; if $k>r, \gamma_{1}(r, k)=u, \gamma_{2}(r, k)=0$; if $k=r, \gamma_{1}(r, k)=\gamma_{2}(r, k)=\min (u, 1), \delta=\min (k, r)$ and $\alpha, \beta$ are nontrivial endomorphisms of $G$ such that $\beta \alpha=\alpha$.

(2) N. R. Reilly [11] has determined a structure theorem equivalent to Theorem 3.1 by different methods. According to his terminology, a bisimple semigroup $S$ is called a bisimple $\omega$-semigroup if $E_{S}$ is integrally ordered. If $E_{S}$ is lexicographically ordered we will call $S$ an $L$-bisimple semigroup.

(3) A bisimple semigroup $S$ is $L_{n}$-bisimple ( $I$-bisimple, $I$ - $\omega$-bisimple) if $E_{S}$ is $n$-lexicographically ordered (is order isomorphic to $Z$ under the reverse of the usual order, is order isomorphic to $Z X I^{0}$ under the usual lexicographic order [Van der Waerden, Vol. 1, p. 81]). We describe the structure of these classes of semigroups completely mod groups in [12], [13], and [16]. The structure theorem for $L$-bisimple semigroups generalizes Theorem 3.2. We investigate several of the properties of $L$-bisimple, $I$-bisimple and $I$ - $\omega$-bisimple semigroups, such as homomorphisms, congruences, and (ideal) extensions in [12], [13], [14], [17], and [18]. The method of attack- initiated here- which readily allows applications of results of [7]-[9] is used throughout.

(4) We will also call the $n$-dimensional bicyclic semigroup the $2 n$-cyclic semigroup in future papers.

(5) We have also studied some of the properties of the semigroups whose structure has been given here in [13] and [15].

\section{REFERENCES}

1. R. H. Bruck, A Survey of Binary Systems, Ergebnisse der Math., Heft 20, Springer, Berlin, 1958. 
2. A. H. Clifford, A class of d-simple semigroups, Amer. J. Math. 75 (1953).

3. A. H. Clifford, and G. B. Preston, The algebraic theory of semigroups, Math. Surveys No. 7, Amer. Math. Soc., Providence, 1962.

4. W. D. Munn, and R. Penrose, A note on inverse semigroups, Proc. Cambridge Philos. Soc. 51 (1955), 369-399.

5. L. Rédei, Die Verallgemeinerung der Schreierschen Erweiterungstheorie, Acta Sci. Math., Szeged 14 (1952), 252-273.

6. D. Rees, On the ideal structure of a semi-group satisfying a cancellation law, Quarterly J. Math. Oxford Ser. 19 (1948), 101-108.

7. R. J. Warne, Matrix representation of d-simple semigroups, Trans. Amer. Math. Soc. 106 (1963), 427-35.

8. - Homomorphisms of $d$-simple inverse semigroups with identity, Pacific J. Math 14 (1964), 1111-1222.

9. - A characterization of certain regular d-classes in semigroups, Illinois J. Math. 9 (1965), 304-306.

10. Regular d-classes whose idempotents obey certain conditions, Duke J. Math. 33 (1966), 187-195.

11. N. R. Reilly, Bisimple w-semigroups, Proc. Glasgow Math. Assoc. 7 (1966), 160167.

12. R. J. Warne, On certain bisimple inverse semigroups, Bull. Amer. Math. Soc. (1966), July.

13. I-bisimple semigroups, to appear.

14. Extensions of I-bisimple semigroups, Canadian J. Math.

15. - A Class of L-bisimple semigroups, to appear.

16. - Bisimple inverse semigroups mod groups, to appear.

17. — L-Bisimple semigroups, to appear.

18. — I- - -Bisimple semigroups, to appear.

Received July 2, 1965.

WEST VIRGINIA UNIVERSITY

MORGANTOWN, WEST VIRGINIA 



\section{PACIFIC JOURNAL OF MATHEMATICS}

\section{EDITORS}

\author{
H. SAMELSON, \\ Stanford University \\ Stanford, California \\ R. M. BLUMENTHAL \\ University of Washington \\ Seattle, Washington 98105
}

\author{
*J. DugundJI \\ University of Southern California \\ Los Angeles, California 90007 \\ RICHARD ARENS \\ University of California \\ Los Angeles, California 90024
}

\section{ASSOCIATE EDITORS}
E. F. BECKENBACH
B. H. NeUmanN
F. WOLF
K. YOSIDA

\section{SUPPORTING INSTITUTIONS}

\author{
UNIVERSITY OF BRITISH COLUMBIA \\ CALIFORNIA INSTITUTE OF TECHNOLOGY \\ UNIVERSITY OF CALIFORNIA \\ MONTANA STATE UNIVERSITY \\ UNIVERSITY OF NEVADA \\ NEW MEXICO STATE UNIVERSITY \\ OREGON STATE UNIVERSITY \\ UNIVERSITY OF OREGON \\ OSAKA UNIVERSITY \\ UNIVERSITY OF SOUTHERN CALIFORNIA
}

\author{
STANFORD UNIVERSITY \\ UNIVERSITY OF TOKYO \\ UNIVERSITY OF UTAH \\ WASHINGTON STATE UNIVERSITY \\ UNIVERSITY OF WASHINGTON \\ * * * \\ AMERICAN MATHEMATICAL SOCIETY \\ CHEVRON RESEARCH CORPORATION \\ TRW SYSTEMS \\ NAVAL ORDNANCE TEST STATION
}

Mathematical papers intended for publication in the Pacific Journal of Mathematics should be typewritten (double spaced). The first paragraph or two must be capable of being used separately as a synopsis of the entire paper. It should not contain references to the bibliography. Manuscripts may be sent to any one of the four editors. All other communications to the editors should be addressed to the managing editor, Richard Arens at the University of California, Los Angeles, California 90024 .

50 reprints per author of each article are furnished free of charge; additional copies may be obtained at cost in multiples of 50 .

The Pacific Journal of Mathematics is published monthly. Effective with Volume 16 the price per volume (3 numbers) is $\$ 8.00$; single issues, $\$ 3.00$. Special price for current issues to individual faculty members of supporting institutions and to individual members of the American Mathematical Society: $\$ 4.00$ per volume; single issues $\$ 1.50$. Back numbers are available.

Subscriptions, orders for back numbers, and changes of address should be sent to Pacific Journal of Mathematics, 103 Highland Boulevard, Berkeley 8, California.

Printed at Kokusai Bunken Insatsusha (International Academic Printing Co., Ltd.), No. 6, 2-chome, Fujimi-cho, Chiyoda-ku, Tokyo, Japan.

PUBLISHED BY PACIFIC JOURNAL OF MATHEMATICS, A NON-PROFIT CORPORATION

The Supporting Institutions listed above contribute to the cost of publication of this Journal, but they are not owners or publishers and have no responsibility for its content or policies.

* Paul A. White, Acting Editor until J. Dugundji returns. 


\section{Pacific Journal of Mathematics}

\section{Vol. 18, No. 3 \\ May, 1966}

William George Bade and Philip C. Curtis, Jr., Embedding theorems for commutative Banach algebras .......................... 391

Wilfred Eaton Barnes, On the $\Gamma$-rings of Nobusawa ................. 411

J. D. Brooks, Second order dissipative operators ................ 423

Selwyn Ross Caradus, Operators with finite ascent and descent ........ 437

Earl A. Coddington and Anton Zettl, Hermitian and anti-hermitian properties of Green's matrices .......................... 451

Robert Arnold Di Paola, On sets represented by the same formula in distinct consistent axiomatizable Rosser theories ................... 455

Mary Rodriguez Embry, Conditions implying normality in Hilbert space ...........................................

Garth Ian Gaudry, Quasimeasures and operators commuting with convolution ................................... 461

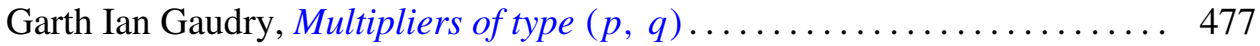

Ernest Lyle Griffin, Jr., Everywhere defined linear transformations affiliated with rings of operators .................................. 489

Philip Hartman, On the bounded slope condition ................ 495

David Wilson Henderson, Relative general position ................ 513

William Branham Jones, Duality and types of completeness in locally

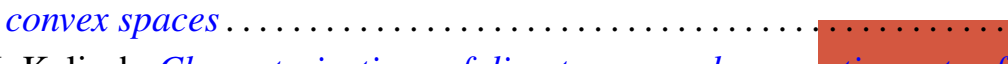

G. K. Kalisch, Characterizations of direct sums and commuting sets of Volterra operators....

Ottmar Loos, Über eine Beziehung zwischen Malcev-Algebren und Lietripelsystemen

Ronson Joseph Warne, A class of bisimple inverse semigroups . . . 